J. Clin. Chem. Clin. Biochem.

Vol. 20, 1982, pp. 181-183

\title{
Direkte Bestimmung von Phenylalanin in Serum mittels Derivativspektrophotometrie
}

Von K.-H. Kullmann

Lehrstuhl für Allgemeine Chemie und Biochemie der Technischen Universität München, Freising-Weihenstephan

\section{W. Endres}

Kinderklinik der Ludwig-Maximilians-Universität München

\section{S. Kirzinger und H.-L. Schmidt}

Lehrstuhl für Allgemeine Chemie und Biochemie der Technischen Universität München, Freising-Weihenstephan

(Eingegangen am 15. Juni/4. Dezember 1981)

Zusammenfassung: Es wird ein einfaches und präzises Verfahren zur Bestimmung von Phenylalanin in Serum- oder Plasmaproben mit Hilfe der Derivativspektrophotometrie beschrieben. $100 \mu l$ Serum oder Plasma werden nach Entfernung von Protein und Harnsäure direkt auf den Gehalt an Phenylalanin analysiert. Dabei ist die Amplitude zwischen dem Peakminimum bei $254 \mathrm{~nm}$ und dem Peakmaximum bei $257 \mathrm{~nm}$ des Derivativspektrums (2. Ableitung eines Absorptionsspektrums) einer Probe dem Gehalt an Phenylalanin direkt proportional. Die Präzision beträgt, in Abhängigkeit von der Konzentration an Phenylalànin, 2,1-10,3\%, die Wiederfindung liegt zwischen 97,1 und $101,1 \%$. Die Empfindlichkeit beträgt $5 \mathrm{mg} / 1$ Serum. Die Methode wurde mit einer säulenchromatographischen Phenylalaninbestimmung verglichen.

\section{Direct determination of phenylalanine in serum by derivative spectrophotometry}

Summary: A simple and precise method is described for the determination of phenylalanine in serum or plasma, using derivative spectrophotometry. After the removal of protein and uric acid, $100 \mu \mathrm{l}$ serum or plasma are analysed directly for phenylalanine. The magnitude of the difference between the peak minimum at $254 \mathrm{~nm}$ and the peak maximum at $257 \mathrm{~nm}$ of the derivative spectrum (2nd derivative of the absorption spectrum) is directly proportional to the phenylalanine content. The precision was $2.1-10.3 \%$, depending on the concentration of phenylalanine. Recovery was between 97.1 and $101.1 \%$. Sensitivity was $5 \mathrm{mg} / 1$ serum. The method was compared with a column chromatographic determination of phenylalanine.

\section{Einfuihrung}

Bestimmungen von Phenylalanin im Serum sind zur Diagnose der Phenylketonurie sowie zur Überwạchung der Diättherapie notwendig. Die Diagnose (Neugeborenen-Screening) erfordert Serienuntersuchungen mit sehr geringem Probenvolumen. Hierfür hat șich der Bakterienhemmtest nach Guthrie \& Susi (1) als geeignet erwiesen. Die Kontrolle der Dịättherapie erfordert exaktere Messungen, die vor allem durch die fluorimetrische Bestimmungsmethode nach McCaman \& Robins (2) sowie die aufwendigere Ionenaustauscher-Säulenchromatographie (3-5) möglich sind. Die hier vorgeschlagene Methode erfordert ein registrierendes Spektralphotometer mit einem Differenzierzusatz. Sie macht sich die verbesserte spektrale Auflösung von Derivativspektren (graphische Darstellung der Steigung eines Spektrums) zunutze
(Abb. 1). Bei der Differenzierung eines Spektrums können Störungen durch Fremdabsorptionen oder Trübungen eliminiert werden $(6,7)$. Daher ist eine schnelle und präzise Bestimmung von Phenylalanin in Serum oder Pिlasma ohne vorherige chromatographische Abtrennung von Phenylalanin und ohne dessen Derivatisierung möglich.

\section{Material und Methoden}

\section{Material}

Phenylalanin, Natriumborat, Natriumcarbonat und Kreatinin wurden von der Fa. Merck, Darmstadt, Uricase von der Fa. Boehringer, Mannheim, bezogen. Ultrafiltrationsmembrankegel erhielten wir von der Fa. Amicon GmbH, Witten. Als Photometer verwendeten wir das Zweistrahlspektralphotometer 555 der Fa. Perkin Elmer, Uberlingen, das mit einem serienmäßigen Differenzierzusatz ausgestattet ist. 

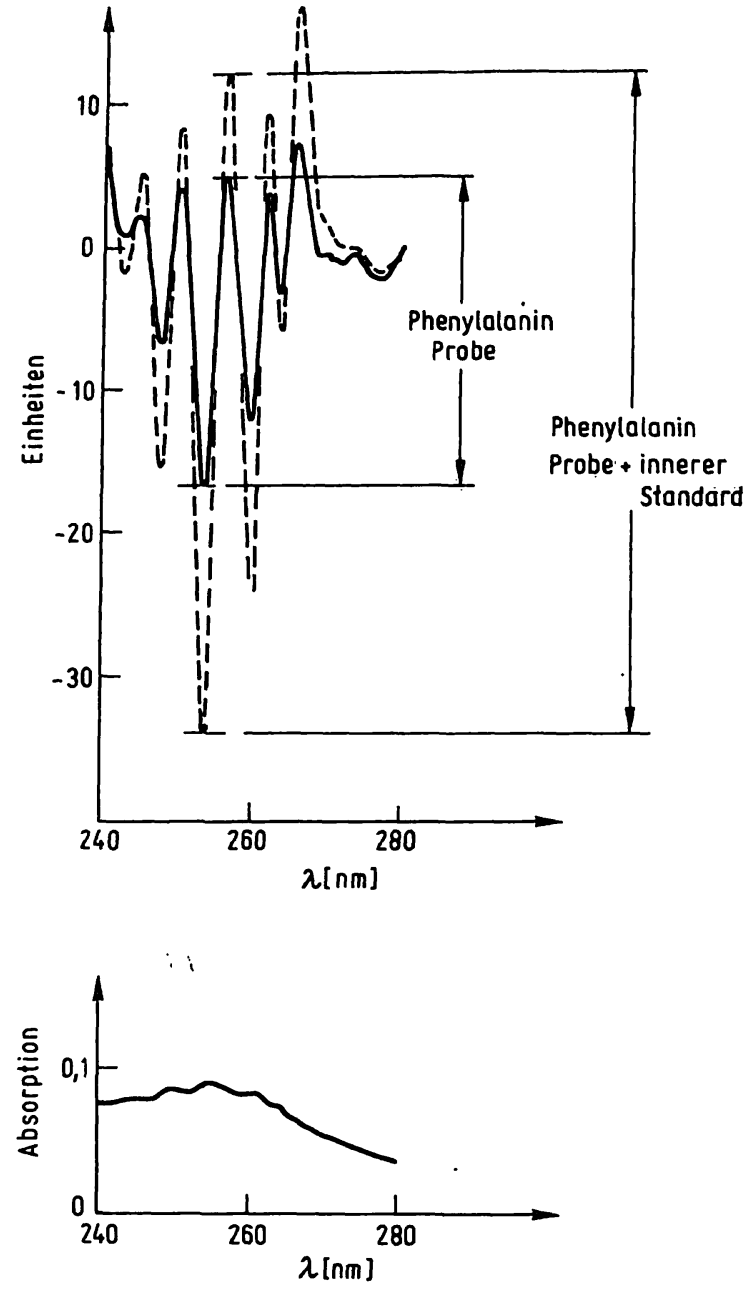

Abb. 1. Absorptionsspektrum (unten) und 2. Ableitung des Absorptionsspektrums (oben) eines Serums mit Phenylalanin.

Der Phenylalaningehalt der Probe wurde auf den Phenylalaningehalt des Inneren Standards $(1,2 \mathrm{mmol} / \mathrm{l})$ bezogen.

\section{Methodik}

$100 \mu 1$ Serum oder Plasma werden mit $900 \mu 15$ mmol/1 Boratpuffer $\mathrm{pH} 8,0$ und $10 \mu \mathrm{l}$ Uricase-Lösung $(2 \mathrm{~g} / \mathrm{l})$ versetzt und $10 \mathrm{~min}$ bei $20^{\circ} \mathrm{C}$ inkubiert. Die Abtrennung des Proteins erfolgt mit gewässerten $\left(\mathrm{H}_{2} \mathrm{O}, 1 \mathrm{~h}\right)$ und vorzentrifugierten $(10 \mathrm{~min}$, $900 \mathrm{~g}$ ) Ultrafiltrationsmembrankegeln (Ausschlußgröße $M_{\mathrm{r}}=25000$ ) in $20 \mathrm{~min}$ bei $900 \mathrm{~g}$. Von $500 \mu \mathrm{l}$ des Filtrates wird in einer $1 \mathrm{~cm}$-Halbmikro-Quarzküvette die 2. Ableitung des Absorptionsspektrums zwischen 250 und 280 gegen eine Referenzlösung ( $5 \mathrm{mmol} / 1$ Boratpuffer mit $10 \mu \mathrm{mol} / 1$ Kreatininlösung) registriert (Photometereinstellung: Spaltbreite $1 \mathrm{~nm}$, Schreiberausschlag Abszisse $10 \mathrm{~nm} / \mathrm{cm}$, Ordinate 0,02 bis - 0,02, Geschwindigkeit $30 \mathrm{~nm} / \mathrm{min}$, Zeitkonstante $10 \mathrm{~s}$ ).

Nach 3maliger Registrierung setzt man der Probe $100 \mu$ Inneren Standard $(0,3 \mathrm{mmol} / 1$ Phenylalanin bzw. $1,2 \mathrm{mmol} / 1$ Phenylalanin) zu und registriert das Spektrum erneut dreimal. Die Abstände zwischen dem Minimum (254 nm) und dem Maximum $(257 \mathrm{~nm})$ des Spektrums sind den Phenylalaninkonzentrationen direkt proportional (Abb. 2). Daher erfolgt die Auswertung über eine „Peak zu Peak-Messung“" (Abstandsdifferenz).

Die Konzentrationen in der Serumprobe $\mathbf{x}(\mathrm{mmol} / \mathrm{l})$ errechnen sich unter Verwendung des Inneren Standards y $(\mathrm{mmol} / \mathrm{l})$ und Berücksichtigung der Verdünnungen nach folgender Formel:

$$
x=y \cdot \frac{a \cdot f_{2} \cdot f_{3}}{b \cdot f_{1}-a} \quad[\mathrm{mmol} / 1]
$$

a = registrierter Schreiberausschlag nach Messung der Probe (rel. Einheiten)

b = registrierter Schreiberausschlag nach Messung der Probe + innerer Standard (rel. Einheiten)

$f_{1} \doteq$ Verdünnungsfaktor für Verdünnung durch Zugabe des inneren Standards

$f_{2}=$ Verdünnungsfaktor für Verdünnung der Serum-Probe

$f_{3}=$ Verdünnungsfaktor für Verdünnung durch Ultrafiltration mit vorgeweichten Membrankegeln

Für den hier beschriebenen Bestimmungsansatz mit $1.00 \mu \mathrm{l}$ Serum oder Plasma ergeben sich folgende Werte:

$y=0,06$ bzw. $0,24 \mathrm{mmol} / 1, f_{1}=1,2, f_{2}=10,1, f_{3}=1,08$

Die Zeit für I Bestimmung beträgt $50 \mathrm{~min}$. Bei gleichzeitiger Bestimmung mehrerer Proben verkürzt sich die Zeit erheblich. Diè verwendeten Lösungen sịnd im Kühlschrank etwa 1 Monạt ștabil.

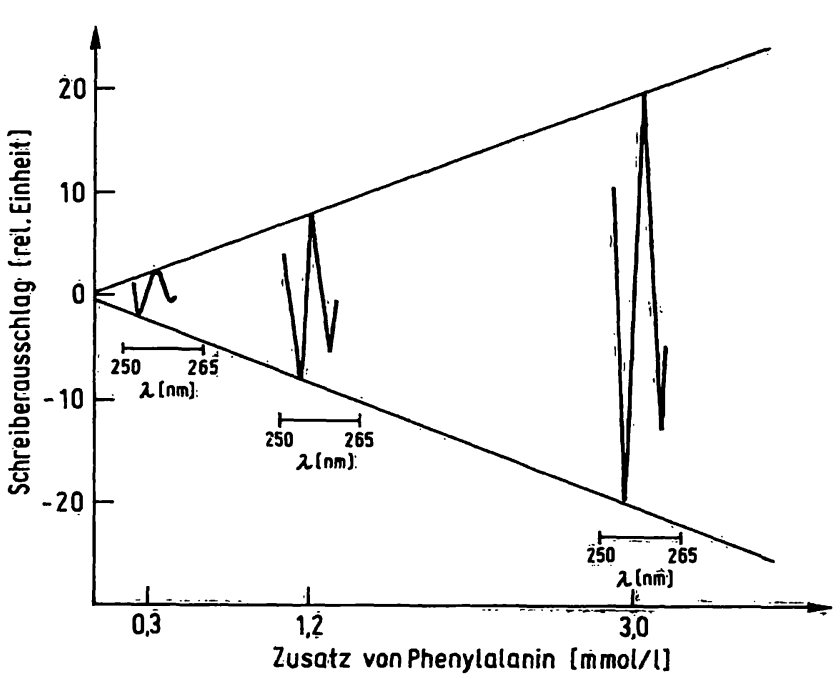

Abb. 2. Schreiberausschlag in Abhängigkẹit von der Konzentration an Phenylalanin im Serum (2. Ableitung des Absorptionsspektrums).

\section{Vergleichsmethode}

Die säulenchromatographische Bestimịung von Phenylalanin erfolgte mit einem Aminosäuren-Autoanàlyzer (LC 2000, Biotronik, München) nach einer weitreichenden Modifikation der von Spackman et al. zur Trennung von Aminosäuren aus physiologischen Flüssigkeiten angegebenen Methode:

Die Trennsäule besteht aus dem Ionenaustauscherharz DC $6 \mathrm{~A}$ (Durrum) mit dèn Ausmaßen 0,6 $\times 20 \mathrm{~cm}$. D̈ie Säulentemperatur steigt zwischen Serin und Asparagin von $36^{\circ} \mathrm{C}$ auf $59^{\circ} \mathrm{C}$ an. Die verwendeten Lithiumcitratpuffer haben die folgenden Ionenstärken und pH-Werte: Puffer A 0,18 mol/1, pH 2,8, Puffer B $0,23 \mathrm{~mol} / 1$, pH 3,1, Puffer C $0,35 \mathrm{~mol} / 1$, pH 3,35, Puffer D $0,40 \mathrm{~mol} / 1, \mathrm{pH} 4,0$, Puffer E 1,65 mol/1, pH 3,3. Diê Pufferdurchflußgeschwindigkeit beträgt $31 \mathrm{ml} / \mathrm{h}$.

\section{Ergebnisse}

\section{Präzision}

Zur Bestimmung der Präzision verwendeten wir ein mit Phenylalanin aufgestocktes Serum. Die Variationskoeffizienten lagen, in Abhängigkeit von der Konzentration an Phenylalanin, zwischen 2,1 und 10,3\% (Tàb. 1).

\section{Rich tigkeit}

Auch zur Bestimmung der Wiederfindung würden mit Phenylalanin aufgestockte Seren verwendet. Die Wiederfindung betrug, in Abhängigkeit von der Konzentration an Phenylalanin, 97,1 bị $101,1 \%$ (Táb. 1). 
Tab. 1. Wiederfindung und Präzision.

\begin{tabular}{|c|c|c|c|c|}
\hline \multirow[t]{2}{*}{$\begin{array}{l}\text { Anzahl } \\
\text { der } \\
\text { Analysen }\end{array}$} & $\begin{array}{l}\text { Phenylalanin } \\
\text { Sollwert }\end{array}$ & Mittelwert & $\begin{array}{l}\text { Wieder- } \\
\text { findung }\end{array}$ & \multirow{2}{*}{$\begin{array}{l}\text { Variations- } \\
\text { koeffizient } \\
\text { (\%) }\end{array}$} \\
\hline & $(\mu \mathrm{mol} / 1)$ & $(\mu \mathrm{mol} / \mathrm{l})$ & $(\%)$ & \\
\hline $\begin{array}{l}19 \\
14 \\
19 \\
18\end{array}$ & $\begin{array}{r}- \\
391,2 \\
1291,2 \\
3091,2\end{array}$ & $\begin{array}{r}91,2 \\
379,8 \\
1272,6 \\
3125,4\end{array}$ & $\begin{array}{r}- \\
97,1 \\
98,6 \\
101,1\end{array}$ & $\begin{array}{r}10,3 \\
4,4 \\
2,1 \\
2,6\end{array}$ \\
\hline
\end{tabular}

\section{Spezifität}

Dank der hohen spektralen Auflösung eines Derivativspektrums ist die Methode sehr spezifisch. Sie wird durch Tyrosin, Tryptophan, Phenylpyruvat und Phenyllactat nicht gestört, da die 2. Ableitung der Spektren dieser Substanzen scharfe und schmale Peaks liefert, die sich mit den zur Auswertung herangezogenen Peaks von Phenylalanin (254-257 nm) nicht überlagern. Hohe Konzentrationen von Harnsäure würden stören, deshalb wurde die im Serum enthaltene Harnsäure vor der Bestimmung mit Uricase abgebaut. Das ebenfalls im Serum enthaltene Kreatinin kann zu einer geringen Störung der Phenylalaninbestimmung führen. Die Referenzküvette enthält deshalb eine annähernd der Konzentration im Serum entsprechende Menge an Kreatinin. Schwankungen des Kreatiningehaltes ịm Normalbereich wirken sich kaum aus (eine Erhöhung des Kreatiningehaltes um $0,2 \mathrm{mmol} / 1$ erniedrigt die gefundenen Phenylalaninkonzentrationen um etwa 1\%). Nur bei pathologisch hohen Kreatininkonzentrationen wäre eine Korrektur durch eine entsprechende Erhöhung des Kreatiningehaltes der Referenžküvette erforderlich. Hohe Kreatininkonzentrationen haben im Absorptionsspektrum eine relativ erhöhte Absorption bei $240 \mathrm{~nm}$ zur Folge. Für störungsfreie Phenylalaninbestimmungen sollte deshalb die Absorption bei $240 \mathrm{~nm}$ kleiner als die bei $260 \mathrm{~nm}$ sein. Andernfalls ist eine Korrektur durch entsprechende Erhöhung des Kreatiningehaltẹ der Referenzküvette angezéigt, wóbei eine exakte Kenntnis des Kreatiningehaltes der Probe nicht erforderlich ist.

\section{Nachweisgrenze}

Die Nachweisgrenze beträgt $3 \mathrm{nmol}$ Phenylalanin (Signal zu Rausch-Verhältnis 3:1). Das sind bei 100 $\mu 1$ Serumprobe $30 \mu \mathrm{mol} / 1$ Phenylalanin.

\section{Literatur}

1. Guthrie, R. \& Susi, A. (1963) Pediatrics 32, 33.8-343.

2. McCaman, M. W. \& Robins, E. (1962) J. Lab, Clin. Med. 59, 885-890.

3. Spackman, D. H., Stein, W. H. \& Moore, S. (1958) Anal. Chem. 30, 1190-1206.

4. Schmidt, G. J., Olson, D. C. \& Slavin, W. (1979) J. Chromatogr. $164,355-362$.

5. Tarbit, I. F., Richardson, J. P. \& Dale, G. (1980) J. Chromatogr. 181, 337-346.

\section{Methodenvergleich}

Ein Vergleich der derivativspektrophotometrisch ermittelten Phenylalaninwerte mit den säulenchromatographisch bestimmten Werten ergab eine gute Ubereinstimmung $(r=0,993)$ beider Methoden (Abb. 3).

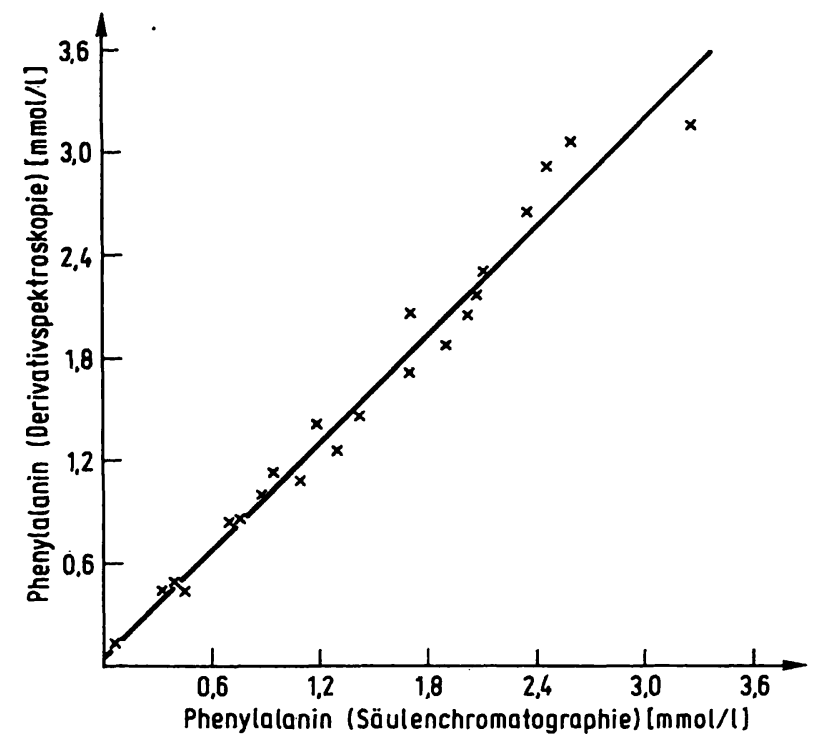

Abb. 3. Vergleich der derivativspektrophotometrischen Phenylalaninbestimmung mit der säulenchromatographischen Methode $(r=0,993, N=22, y=1,048 x+0,774)$.

\section{Diskussion}

Das beschriebene Verfahren stellt eine einfache, schnelle und präzise Methode zur Bestimmung von Phenylalanin in Serum oder Plasma dar. Der Vorteil gegenüber gängigen fluorimetrischen und chromatographischen Verfahren liegt bei gleicher Richtigkeit und Präzision im geringen apparativen und zeitlichen Aufwand begründet. Deshalb ist die Methode vor allem zur schnellen Kontrolle der diätetischen Behandlung von Phenylketonurie-Patienten geeignet.

Die Derivativspektrophotometrie wurde bereits mit Erfolg zur Bestimmung von aromatischen Aminosäuren in Bakteriensuspensionen, zellfreien Extrakten und Proteinen eingesetzt (7-9). Auch zur Untersuchung von Humanșeren könnte eine gleichzeitige derivativspektrophotometrische Bestimmung von Phenylalanin, Tyrosin und Tryptophan von Interesse sein. Zu prüfen wäre auch, inwieweit sich andere Serumkomponenten sowie Komponenten von Speichel und Urin mit Hilfe der Derivativspektrophotometrie erfassen ließen.

6. Talsky, G., Mayring, L. \& Kreuzer, H. (1978) Angew. Chemie 90, 840-854.

7. Kullmann, K.-H. (1980) Forum Mikrobiologie 5, 304-306.

8. Ichikawa, T. \& Terada, H. (1979) Biochim. Biophys. Acta 580, 120-128.

9. Matsushima, A., Inouc, Y. \& Shibata, K. (1975) Anal. Biochem. 65, 362-368.

Dr. K.-H. Kullmann Prcysingstr. 3

D-8309 Au i.d. Hallertau 
。 


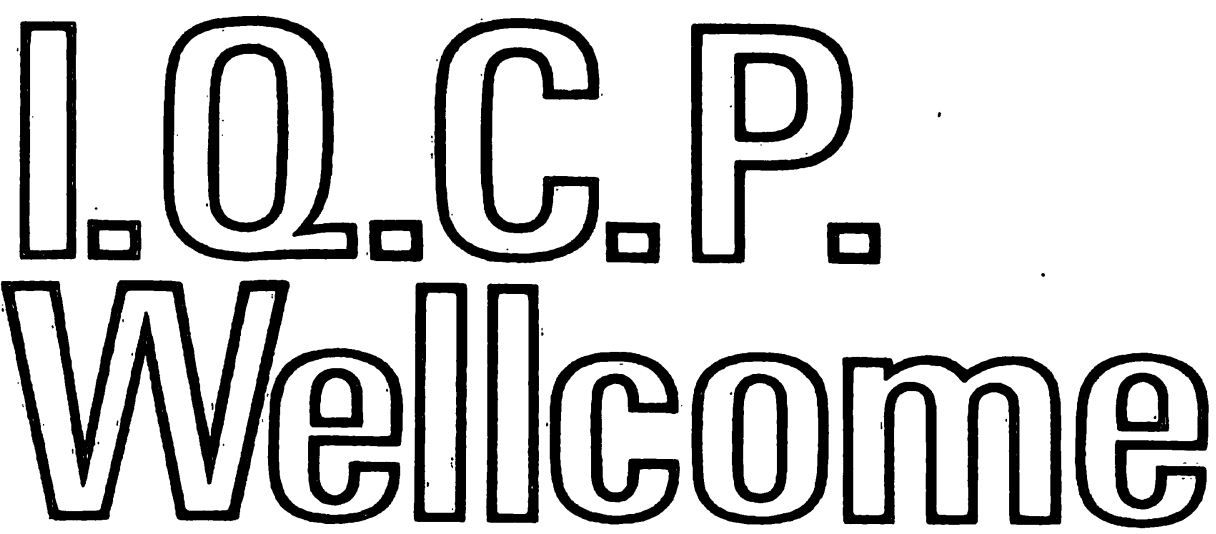

Das Immunoassay Qualitätskontrollprogramm bewertet Ihre Immunoassay-Ergebnisse im internationalen Vergleich anhand von 16 Parametern in zwei jährlichen Zyklen

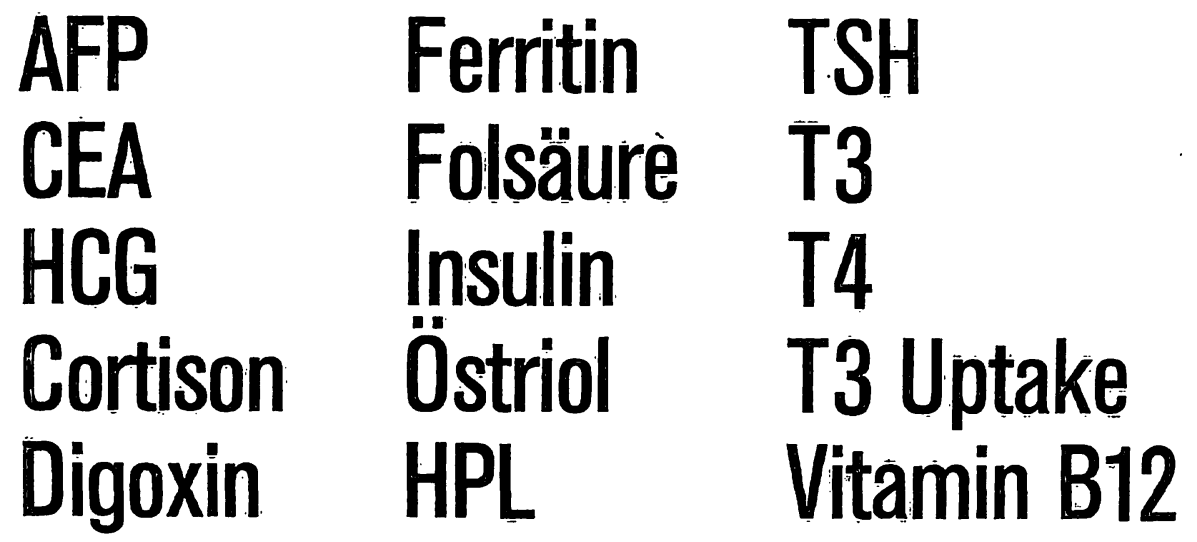

Deutsche Wellcome GmbH

Abteilung Diagnostica

Postfach 109

3006 Burgwedel 1

Telefon (05139) 3001

Telex 0922799 welco d

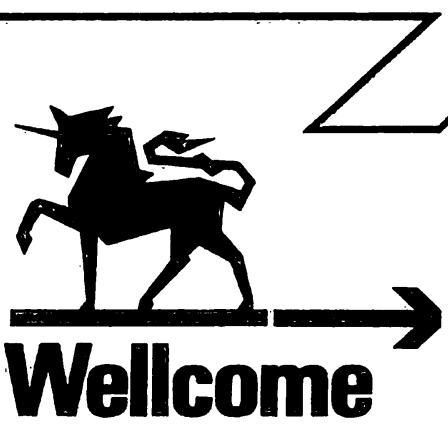

Dỉagn@Sțca 

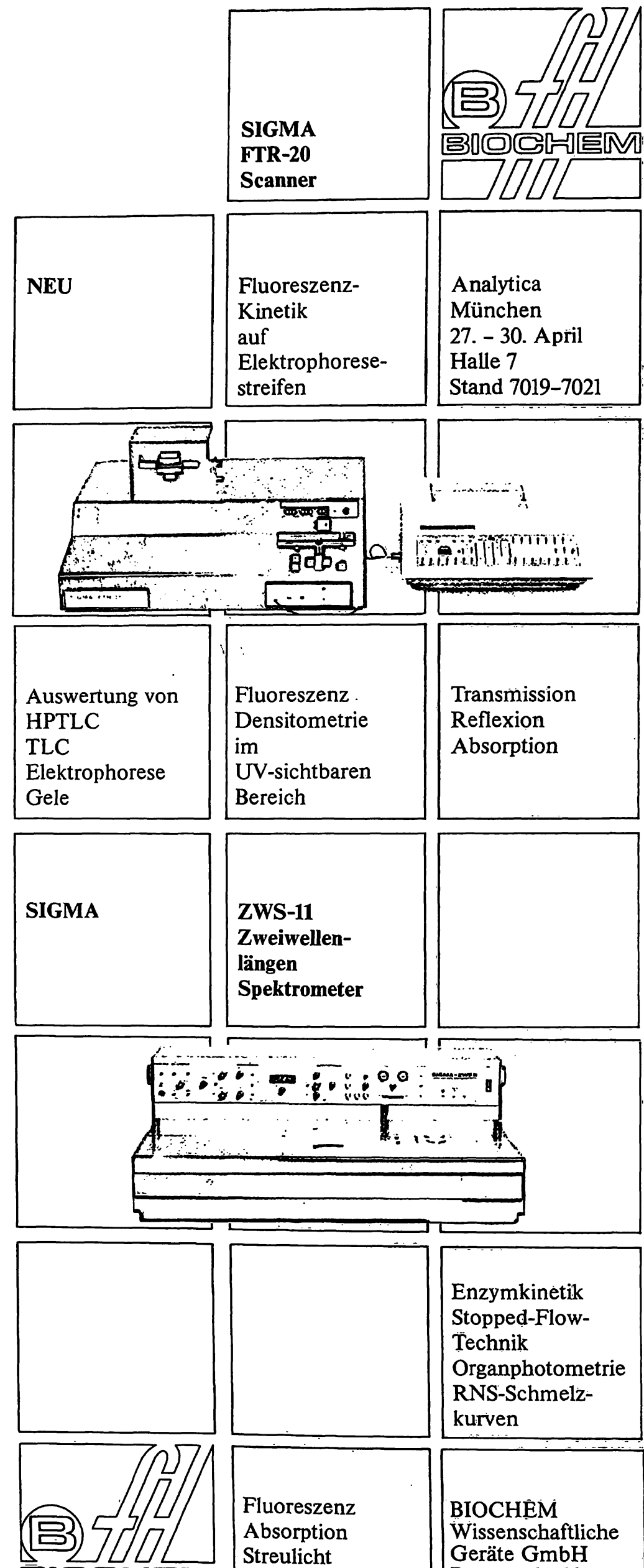

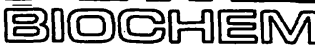

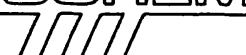

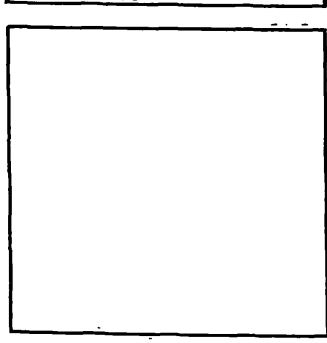

Fluoreszenz Absorption Streulicht Luminiszenz
Enzymkinetik Stopped-FlowTechnik Organphotometrie RNS-Schmelzkurven

BIOCHEM Wissenschaftliche Geräte $\mathrm{GmbH}$ Benzstraße 28 D-8039 Puchheim Tel. 089-806081

\section{Survey of Drug Research in Immunological Disease \\ Vassil St. Georgiev, Rochester, N.Y.}

Vol. 1: Aliphatic Derivatives

ca. 600 p., 4 tab., 1982. ca. SFr, 490.- / DM 588.ISBN 3-8055-3503-1

\section{Theilheimer's Synthetic Methods of Organic Chemistry}

Series Editor: A.F. Fịnch, Dẹrwent Publications Ltd., Londón

Vol. 36: Yearbook 1982

ca. 510 p., 1982. ca. SFr. 498.=/ DM 597.=

ISBN 3-8055-3446 $=9$

\section{Einfựhruing in die}

\section{praktische Biochemie}

fưr Studierende der Mediżin, Vètẹinărmẹediz̄in, Pharmạazie und Biologie

3., vollstăndig neu bearbeitete Auflage

H. Aebi, Bern

ca. 410 S., 1982. ca. SFr./DM 60.-

ISBN 3-805 5-3448-5

\section{Interpretation klinisch-chemischer \\ Laborresultate}

2., nẹu bearbeitete und ervieiterte Aufläge

R.D. Eastham, Bristol

übersetzt und bearbeitet von E. Peheim und J.P. Cọlọmbịọ,

Bern

XIV + 262 S. 1981. SFr./DM 29.-

ISBN 3-8055-1879-X

\section{Klinische Chemie:}

Theorie, Praxis, Interpretation

4., vollstăndig neu bearbeitete Auflage

Herausgeber: R. Richterich $t$ und J.P. Colombo, Bern

XVI + 620 S., 161 Abb., 137 Tab., 1978

SFr./DM'98.-

ISBN 3-8055-2796-9

\section{Chemie im Laboratorium}

Einführung in die allgemeinen theoretischen Grundlagen mit Einblick in die klinische Chemie und Biochemie

3., erweiterte Auflage

K. Lauber, Liebefeld

VIII + 372 S., 104 Abb., 41 Tab., 197.5. SFr./DM 67:-

ISBN 3-8055-2102-2

Bestellungen und

Prospektanfragen richten Sie

bitte an Ihre Buch handlung

odèr an:

S. Karger AG.

Postfach, CH-4009'Bàsel

S. Karger Gimbih,

Postfach 2,

D=8034 Germering/München

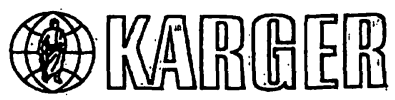

